\title{
HERRAMIENTAS GERENCIALES: USOS, BENEFICIOS Y DIFICULTADES EN SU IMPLEMENTACIÓN EN ORGANIZACIONES DE ECONOMİA POPULAR Y SOLIDARIA
}

\author{
MANAGEMENT TOOLS: USES, BENEFITS AND \\ DIFFICULTIES IN ITS IMPLEMENTATION IN SOCIAL AND \\ SOLIDARITY-BASED ECONOMY ORGANIZATIONS
}

\section{Rocío León}

Magister en Gestión Empresarial, Universidad Técnica Particular de Loja (Loja/Equador).

Profesor Universidad Técnica del Norte (Ibarra/Equador).

E-mail: rgleon@utn.edu.ec

\section{Mónica Gallegos}

Master en tecnologías para la gestión y práctica docente, Pontifica Universidad Católica del Ecuador (Quito/Equador).

Profesor Universidad Técnica del Norte (Ibarra/Equador)

E-mail: mgallegos@utn.edu.ec

\section{Marcelo Vallejos}

Magister en Contabilidad y Auditoría, Universidad Técnica del Norte (Ibarra/Equador).

Profesor Universidad Técnica del Norte (lbarra/Equador).

E-mail: hmvallejos@utn.edu.ec

\section{Wilma Guerrero}

Magister en Gestión y Desarrolo Social, Universidad Técnica Particular de Loja (Loja/Equador).

Profesor Universidad Técnica del Norte (Ibarra/Equador).

E-mail:wmguerrero@utn.edu.ec

\section{Byron Acosta}

Doctor en Administración Universidade Federal do Rio Grande do Sul (Porto Alegre/Brasil).

Profesor Escuela Politécnica Nacional (Quito/Ecuador)

E-mail: byron.acosta@epn.edu.ec

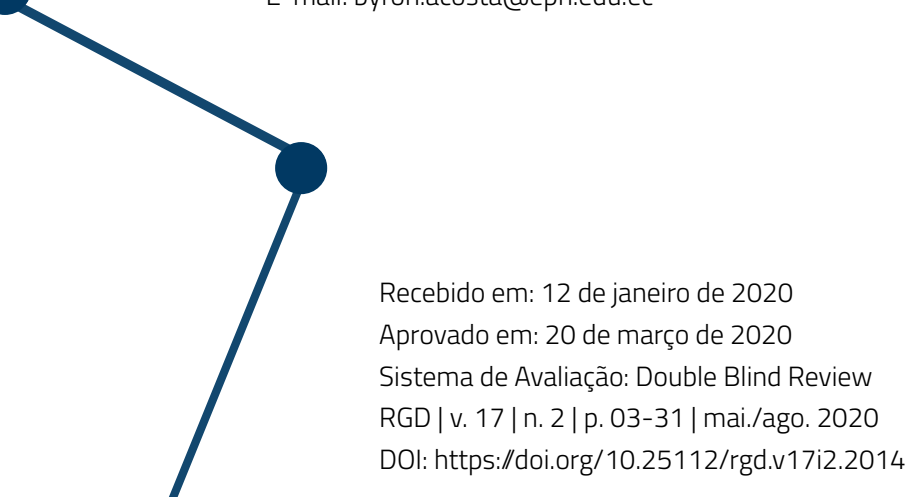




\section{RESUMEN}

El objetivo de esta investigación es determinar los tipos de herramientas gerenciales, sus beneficios y dificultades de adopción en las empresas. Para ello fueron encuestadas 60 organizaciones de economía popular y solidaria de los sectores de comercio, manufactura y servicios. Los resultados muestran que el análisis financiero, la planificación estratégica, la lluvia de ideas y el FODA, son las herramientas de gestión más utilizadas por este tipo de organizaciones. A diferencia de lo encontrado en la literatura, el FODA no ocupa la primera posición de preferencia y uso. En relación a los beneficios de implementación de este tipo de instrumentos, estas organizaciones las usan para la detección de nuevas oportunidades de negocio, la solución de problemas estratégicos y el desarrollo de procesos de mejora continua. Además, las dificultades en su implementación fueron la falta de recursos financieros, falta de tiempo para capacitación y bajo nivel de conocimientos en áreas de tecnología.

Palabras-clave: Herramientas gerenciales. Beneficios empresariales. Barreras de implementación.

\section{ABSTRACT}

The objective of this research is to determine the types of management tools, their benefits and adoption difficulties. Sixty social and solidarity-based economy organizations of commerce, manufacturing and services were surveyed. The results show that financial analysis, strategic planning, brainstorming and SWOT are the most used. Unlike what is found in the literature, the SWOT does not occupy the first position in preference and use. The benefits of its implementation were the detection of new business opportunities, the solution of strategic problems and the development of continuous improvement processes. In addition, the difficulties in its implementation were the lack of financial resources, lack of time for training and low level of knowledge in technology areas.

Keywords: Management tools. Business benefits. Implementation barriers. 


\section{INTRODUCCIÓN}

Las organizaciones, además de poseer estrategias correctamente definidas, necesitan métodos, técnicas, procedimientos y conocimientos para cumplir con sus objetivos de corto, mediano y largo plazos. Según Phaal, Farrukh, \& Probert (2006) existen diferentes herramientas, técnicas, procedimientos, modelos y frameworks para gestionar eficientemente las empresas. En promedio, el número de herramientas utilizadas por las empresas son 17 (CETINDAMAR; WASTI; BEYHAN, 2012). La aplicación de tal o cual herramienta dependerá del tipo de organización y del problema a resolver (QEHAJA; KUTLLOVCI; PULA, 2017). No obstante, las herramientas de gestión deben cumplir tres condiciones fundamentales. Primero, ser prácticas, necesarias para apoyar las decisiones y acciones de gestión; segundo, ser procedimentales, para combinar herramientas y técnicas que aborden problemas comerciales específicos; y tercero, ser frameworks, para guiar en el análisis de gestión de tecnología, basados en principios teóricos bien fundamentados (PHAAL; FARRUKH; PROBERT, 2006).

Independiente del tipo de herramienta que la empresa implemente, sea de gestión, de calidad u otras; tal adopción impactará positivamente en el desempeño y eficiencia de la organización (QEHAJA, 2017). Según Ahmed y Hassan (2003), las empresas que utilizan herramientas de gestión de calidad por un amplio período de tiempo pueden obtener mejor desempeño que aquellas con menos tiempo de implementación. Sin embargo, la cantidad de herramientas implementadas no es un factor que optimice el desempeño en la empresa; como lo indican Cetindamar et al.,(2012) , no se observa una relación entre la rentabilidad de la empresa y el número de herramientas implementadas. Es decir, las empresas no deben adoptar herramientas por moda o por el simple hecho de pensar que a mayor cantidad, mejor resultado. Es necesario evaluar si la empresa está en capacidad de acoger esta nueva tecnología en función de las habilidades y conocimientos de sus funcionarios (CETINDAMAR; WASTI; BEYHAN, 2012).

Investigaciones sobre herramientas gerenciales se han focalizado en temas como los tipos de herramientas más usados por las empresas, los motivos para su adopción, los problemas en su implementación y uso, y los beneficios generados en las firmas. Por ejemplo, Ahmed \& Hassan (2003) investigaron el estado de aplicación de herramientas de gestión de calidad en pequeñas y medianas empresas; los resultados revelaron que la falta de análisis metódico en la aplicación de las herramientas es una debilidad en ese tipo de empresas. Phaal et al. (2006) analizaron la practicidad y la aplicación de herramientas de gestión y tecnológicas; en esa misma línea Nedelko, Potocan, \& Dabić (2015) examinaron las relaciones entre el uso actual y futuro de herramientas gerenciales. Cetindamar et al. (2012a) estudiaron 52 empresas turcas de electrónica y maquinaria para determinar qué herramientas de gestión tecnológica se utilizaban en la práctica, qué determinaba su uso y si afectaba el 
rendimiento de los usuarios. Los resultados mostraron que existe una relación significativa y lineal entre el grado en que las empresas han alcanzado sus objetivos de crecimiento y el número de herramientas y técnicas utilizadas; sin embargo, en esta relación no se observa la rentabilidad de la empresa ni la cantidad de herramientas y técnicas usadas.

Boikanyo, Lotriet, \& Buys (2016) investigaron en qué medida se utilizan herramientas de gestión en 300 empresas de sector minero de África del Sur; los resultados indican que más de la mitad de los encuestados no tienen herramientas de gestión que proporcionen información en tiempo real para el desarrollo de sus actividades. Pawliczek et al. (2015) analizaron el impacto económico de 15 herramientas gerenciales en 677 empresas checas y eslovacas; los resultados mostraron que Kaisen, Six Sigma, MBO y Lean Management, son las herramientas que más influyen positivamente en el impacto económico en las empresas. Fanelli, Lanza, \& Zangrandi (2017) investigaron cómo la adopción de herramientas de gestión mejora el rendimiento de la calidad en la atención médica de hospitales italianos; los resultados indican que la aplicación de herramientas de gestión de calidad sirven para mejorar la profesionalidad clínica de los hospitales.

Qehaja (2017) y Qehaja et al. (2017) mediante un meta-análisis del uso de herramientas de gestión del periodo 1990 a 2015, concluyeron que las herramientas más usadas son: análisis FODA, Benchmarking, análisis PEST, análisis "What if', establecimiento de Visión y Misión, análisis de Porter, análisis financiero de negocios, análisis de factores críticos de éxito, análisis de costo-beneficio y satisfacción de consumidor. Nouri \& Soltani (2017) investigaron las herramientas de gestión más importantes en empresas iraníes en relación a su uso, importancia y satisfacción; los resultados indican que las herramientas más usadas son: establecimiento de la Misión y Visión, análisis FODA, análisis costo-beneficio, Core Competences, análisis financiero, factores críticos de éxito, TQM, análisis de precios, análisis de Stakeholders y Benchmarking.

Precisamente existe una gran variedad de técnicas y herramientas de gestión que las organizaciones pueden adoptar. La decisión de adopción estará condicionada en función de: a) el tamaño de empresa, b) la actividad que desempeña, c) el problema a resolver, d) el grado de implementación -toda la organización o en un área específica-, e) el nivel de capacidades que posee -de conocimiento y económicas-, f) el nivel de compromiso de los miembros de la empresa, y g) el tipo de beneficio esperado (AHMED; HASSAN, 2003). Existen pocas evidencias en la literatura que discuten respecto a los patrones de uso de herramientas de gestión en diferentes áreas de la empresa y examinan las razones para las diferencias en el uso (NEDELKO; POTOCAN; DABIĆ, 2015). En esa misma línea, Qehaja et al. (2017) consideran que las investigaciones relacionadas con el uso de herramientas de gestión estratégica no han sido suficientemente discutidas por académicos y gestores. 
En base a ese contexto, el objetivo de esta investigación es identificar los tipos de herramientas gerenciales más utilizadas por las organizaciones que forman parte del sistema de la economía popular y solidaria, concentrando nuestro estudio en aquellas organizaciones donde sus integrantes, individual o colectivamente, organizan y desarrollan procesos de producción, intercambio, comercialización (...) y consumo de bienes y servicios, para satisfacer necesidades y generar ingresos, basadas en relaciones de solidaridad, cooperación y reciprocidad, privilegiando al trabajo y al ser humano como sujeto y fin de su actividad, orientada al buen vivir, en armonía con la naturaleza, por sobre la apropiación, el lucro y la acumulación de capital (ASAMBLEA NACIONAL DEL ECUADOR, 2014). El estudio de este tipo de organizaciones es importante porque en el Ecuador se evidencia su rol de inclusión económica y social, así como su carácter distributivo y redistributivo. Entre el 2012 y 2016, el número de organizaciones, tanto del sector financiero como del sector no financiero, registradas en la SEPS pasó de 6.016 a 9.139, es decir un crecimiento del $52 \%$, cubriendo todo el territorio nacional con la presencia de más de 214.000 socios o asociados en el sector no financiero y cerca de 5 millones en el sector financiero, que tomaron la opción de compartir sus valores y principios, y de ejercer, en el mismo, su derecho al trabajo y a mejorar sus condiciones de vida, los datos demuestran, no solo la capacidad de inclusión económica social, sino también, la de distribución y redistribución de la riqueza (JACOME et al., 2016). Además, es importante comprender que si bien las herramientas y técnicas son esenciales para gestionar profesionalmente cualquier empresa pequeña, mediana o grande Ahmed \& Hassan (2003), no es igual el control y la gestión de una propiedad familiar, donde la propiedad se transfiere de generación en generación y con el capital físico se transmite la forma histórica la cultura de la gestión y los controles a una empresa constituida como sociedad (HABERKAMP et al., 2019).

Las empresas en su mayoría corresponden a un sector de empresas asociativo es decir constituidas por personas naturales con actividades económicas productivas de similares características que solidarizan su aportación de acuerdo a sus capacidades, bienes, tecnologías y recursos financieros; con un tiempo de vida mayor a 3 años en el mercado, pertenecientes a los sectores de comercio, manufactura y servicios.

\section{REVISIÓN DE LA LITERATURA}

\subsection{TIPOS DE HERRAMIENTAS GERENCIALES}

En las empresas, sus miembros toman decisiones y realizan actividades que tienen diferente nivel de impacto dependiendo de su grado jerárquico y el área a la cual pertenecen. Para que esas actividades 
y decisiones tengan el mínimo riesgo e incertidumbre en el momento de su ejecución, las organizaciones necesitan apoyarse en técnicas o herramientas que faciliten su aplicación. Para Nedelko et al. (2015), las herramientas de gestión engloban un conjunto de conceptos, procesos, ejercicios y frameworks analíticos que van desde la reestructuración total del negocio hasta la implementación de mecanismos de evaluación, seguimiento y medición, que les permiten conocer sus debilidades, fortalezas, oportunidades y amenazas, para así desarrollar estrategias de mejoramiento. Una herramienta de gestión puede ser definida como un conjunto de instrumentos que soportan la implementación de conceptos e ideas en todos los niveles de la empresa, con el objetivo de apoyar en los procesos organizacionales (NEDELKO; POTOCAN; DABIĆ, 2015). Cáceres, Saavedra, Quintero, \& Molina (2004) consideran que el objetivo de las herramientas gerenciales es generar técnicas y destrezas para obtener información pertinente con criterios de calidad, excelencia, creatividad y efectividad, que se conjugan con las estrategias para garantizar su aplicación. Por tanto, el propósito de las herramientas de gestión estratégica y técnicas es ofrecer ganancias y beneficios significativos para la empresa y para contrastar esto, existen pruebas de que las herramientas de gestión estratégica y técnicas pueden hacer una diferencia (PASANEN, 2011).

Las herramientas de gestión se utilizan en actividades, procesos, áreas específicas o en toda la organización con la finalidad de optimizar el uso de recursos para volverse más competitivas. De hecho, con el pasar del tiempo, las técnicas de gestión han proliferado permitiendo mejorar el desempeño, monitorear y evaluar resultados tornándose más sofisticadas y necesarias en el entorno empresarial (FANELLI; LANZA; ZANGRANDI, 2017). Aunque las herramientas sean sofisticadas, para Phaal et al. (2006) estas deben cumplir con los siguientes parámetros: a) fundamentadas en un modelo objetivo de mejores prácticas; b) flexibles, permitiendo el mejor ajuste a la situación actual y a las necesidades de la empresa; c) no mecanicistas o prescriptivas; d) capaces de integrarse con otras herramientas, procesos y sistemas; e) dar como resultado una mejora cuantificable; y f) apoyar la comunicación.

Armstrong (2001) afirma que las herramientas y técnicas gerenciales pueden ser usadas en diferentes ámbitos de la gestión empresarial, por ejemplo: 1) gestión general, 2) gestión del marketing, 3) gestión de operaciones, 4) gestión financiera, 5) gestión de recursos humanos, 6) tecnología de información, 7) gestión de la ciencia y la tecnología, 8) planificación y asignación de recursos, y 9) productividad y eficiencia. Para Cetindamar et al. (2012a) el número promedio más alto de herramientas se usan en actividades de I+D, en estrategia de tecnología y en gestión del conocimiento. La innovación es considerada como una importante estrategia de crecimiento para empresas en América del Norte y Europa (DE OLIVEIRA CARLOS et al., 2015). Sin relación directa con el tipo de herramienta y el área en que sea utilizada, su objetivo fundamental es apalancar la toma de decisiones con el fin de mejorar el desempeño de la empresa. 
Como la literatura lo detalla, existen diversos tipos de herramientas de gestión; indistintamente del tipo de instrumento a implementar. Cetindamar et al. (2012a) las clasifican en seis grupos en función de los objetivos a cumplir en una empresa: 1) herramientas para analizar información externa (Ejemplo: Technology forecasting, benchmarking); 2) herramientas para analizar información interna (Ejemplo, auditorias de innovación); 3) herramientas para calcular la carga de trabajo y los recursos necesarios en los proyectos (Ejemplo: Project management, portafolio de proyectos); 4) herramientas para gestionar el trabajo colaborativo (Ejemplo: Redes de colaboración); 5) Técnicas para la creación de ideas y solución de problemas (Ejemplo: Análisis de valor y creatividad); y 6) herramientas relacionadas con la mejora de la eficiencia y la flexibilidad (Ejemplo: Pensamiento lean, mejoramiento continuo).

Herramientas de gestión estratégica es el nombre genérico que se asigna a cualquier método, modelo, técnica, herramienta, tecnología, framework, metodología o enfoque usado para facilitar la estrategia de trabajo y la toma de decisiones (QEHAJA; KUTLLOVCI; PULA, 2017). Para Pawliczek, Meixnerova, \& Navratilova (2015), existe una amplia variedad de herramientas gerenciales, entre las que se destacan: la matriz BCG, BSC, el modelo EFQM, ISO 9000, ISO 14000, Kaisen, KPI, Lean Management, análisis PEST, las cinco fuerzas de Porter, Six Sigma, SMART, análisis FODA y TQM entre otras. En ese sentido, las herramientas de gestión ayudan a reducir el riesgo e incertidumbre en la toma de decisiones, sin importar el nivel jerárquico de quién las aplica. Además, considerando que los cambios tecnológicos continuamente crean nuevos retos y oportunidades para el desarrollo de nuevos productos, procesos, prácticas organizacionales y de diversificación industrial; se necesita que las oportunidades sean capturadas y convertidas en valor a través de herramientas de gestión tecnológica (CETINDAMAR; WASTI; BEYHAN, 2012).

La Tabla 1. presenta las 10 herramientas de gestión más utilizadas según el nivel de desarrollo de los países. Independientemente del nivel de desarrollo económico del país las herramientas más usadas son: El análisis FODA y las cinco fuerzas competitivas de Porter (QEHAJA, 2017; QEHAJA; KUTLLOVCl; PULA, 2017). En el caso del FODA, los tres grupos de países lo consideran como la herramienta más utilizada -primera posición; en cuanto al análisis de Porter la prioridad de uso es diferente: en octava posición en los desarrollados, en quinta en los países en desarrollo, y en tercera en las economías en transición. Es importante resaltar que la planificación estratégica solo aparece dentro del top 10 en los países en desarrollo. Para Qehaja (2017) y Qehaja et al. (2017) las herramientas más usadas en países desarrollados y en desarrollo son Benchmarking, factores críticos de éxito y análisis costo-beneficio. 


\section{Gestãoe \\ Desenvolvimento}

e-ISSN: 2446-6875

p-ISSN: 1807-5436

Tabla 1. Técnicas de gestión estratégica más usadas por nivel de desarrollo del país*

\begin{tabular}{llll}
\hline \multicolumn{2}{l}{ Países desarrollados } & \multicolumn{1}{c}{ Países en desarrollo } & Economías en transición \\
\hline 1. Análisis FODA & 1. Análisis FODA & 1. Análisis FODA \\
2. Benchmarking & 2. Análisis financiero del negocio & 2. Métodos estadísticos y \\
3. Key competencies & 3. Análisis PEST & matemáticos \\
4. CUSAT & 4. Benchmarking & 3. Análisis de Porter \\
5. Factores críticos de éxito & $\mathbf{5 .}$ Análisis de Porter & 4. Análisis de cadena de \\
6. Análisis de precios & 6. Factores críticos de éxito & & valor \\
7. Costo-beneficio & 7. Análisis financiero de la competencia & & \\
8. Análisis de Porter & 8. Análisis de los Stakeholders & \\
9. Análisis PEST & 9. Planificación estratégica & \\
10. Análisis "What if" & 10. Análisis costo-beneficio & \\
\hline
\end{tabular}

\section{Fuente: Qehaja et al. (2017)}

*Orden descendente de utilización.

En el meta-análisis realizado por Qehaja et al., el análisis FODA es la única técnica usada en todos los tipos de empresas, indiferentemente de su tamaño, y se encuentra en la primera posición del ranking de las más aplicadas (Tabla 2). Según Cetindamar; Wasti; Beyhan (2012a) existe una relación positiva y estadísticamente significativa entre la cantidad de herramientas adoptadas y el tamaño de la empresa; es decir, empresas grandes (en términos de número de empleados) utilizan un número mayor de herramientas que las pequeñas.

Tabla 2. Técnicas de gestión estratégica más usadas por tamaño de empresa*

\begin{tabular}{lll}
\hline \multicolumn{1}{c}{ Grandes } & \multicolumn{1}{c}{ Medianas y Pequeñas } \\
\hline 1. Análisis FODA & 1. Análisis FODA \\
2. Aplicaciones de hojas de cálculo & 2. Análisis PEST \\
3. Análisis de escenarios & 3. Elaboración de presupuestos \\
4. BSC & 4. Benchmarking \\
5. Análisis financiero de la competencia & 5. Establecimiento de Visión y Misión \\
6. Análisis de riesgo & 6. Análisis financiero del negocio \\
& 7. Planificación estratégica \\
& 8. Estrategias de negocio \\
& 9. Escenarios pesimistas y optimistas \\
& 10. Análisis costo-beneficio \\
\hline
\end{tabular}

Fuente: Qehaja et al. (2017)

*Orden descendente de utilización.

El diseño de una herramienta y su aplicación pueden variar considerablemente según el tamaño de la empresa, el tipo (con fines de lucro o sin fines de lucro) y según el propósito (ya sea para inspirar una estructura de pensamiento o simplemente para proporcionar legitimidad) (KNOTT, 2006). Las investigaciones muestran que existen diferencias de uso según el tamaño de las empresas, el sector 


\section{Gestãoe \\ Desenvolvimento}

e-ISSN: 2446-6875

p-ISSN: 1807-5436

de actuación y el nivel de desarrollo del país. Las 10 técnicas más utilizadas alrededor del mundo son (QEHAJA, 2017; QEHAJA; KUTLLOVCI; PULA, 2017) (Figura 1): Análisis FODA, Benchmarking, análisis PEST, análisis "What if', establecimiento de la Visión y Misión, Análisis de Porter, análisis financiero de la empresa, factores críticos de éxito, análisis costo-beneficio, Customer satisfaction (CUSAT). Qehaja (2017), afirma que las cuatro herramientas más usadas de 2000-2014 son: Benchmarkig, Planificación estratégica, Outsourcing, y establecimiento de la Visión y Misión.

Figura 1. Herramientas más utilizadas por las empresas

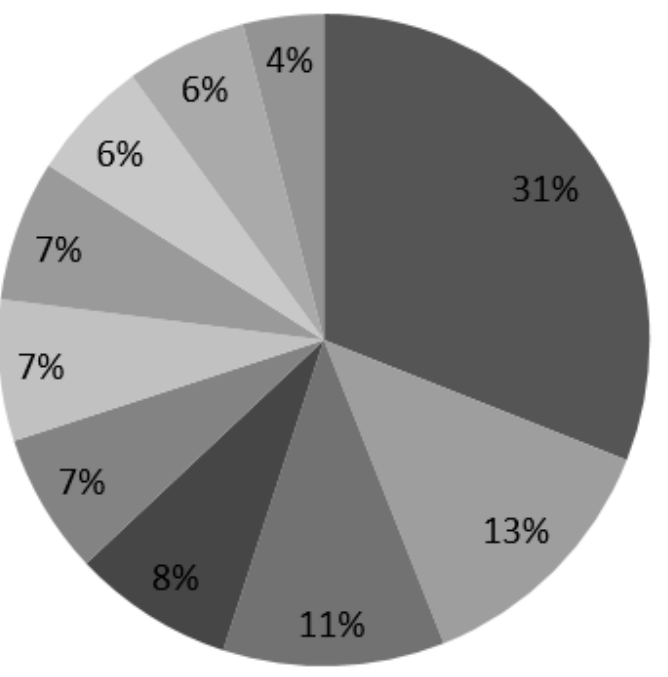

-FODA

- Benchmarking

PEST

"What if"

- Visión y Misión

Analisis de Porter

Análisis financiero

Factores criticos

Costo-beneficio

CUSAT

\section{Fuente: Qehaja et al. (2017)}

De acuerdo a Nouri \& Soltani (2017) el ranking de modelos y herramientas de gestión más usados por las empresas son: Customer Relationship Management/CRM, Benchmarking, Evaluación de la participación de los empleados, Planificación estratégica, Outsoursing, Balanced Scorecard (BSC / Cuadro de Mando Integral), Establecimiento de Visión y Misión, Gestión de la cadena de suministros, Segmentación del mercado, Análisis de metadata, Gestión del cambio, Core competency, Total Quality Management / TQM, Fusiones y adquisiciones, Reingeniería, Satisfacción y gestión de la lealtad, Alianza estratégica, Gestión del tiempo, Transformación digital, Contingencia y planificación de escenarios, Reducción de la complejidad, Modelos de optimización de precios, Zero-based budgeting, Innovación disruptiva. De todas 
las herramientas listadas, la planificación estratégica es la más popular y efectiva en América del Norte y del Sur, sin embargo, su aplicación es reducida en Asia y Europa (NOURI; SOLTANI, 2017).

Según el tipo de industria, solo existen diferencias en el uso del costeo ABC, BSC, análisis costobeneficio, análisis del ciclo de vida del producto, análisis de la cadena de suministros, equipos virtuales, entre las empresas de manufactura y servicios (NOURI; SOLTANI, 2017). Según el tamaño, solo existen diferencias en el uso del BSC, análisis de introducción de nuevo producto, análisis PEST, planificación de escenarios, alianzas estratégicas, análisis de la cadena de suministros y teoría de juegos, entre empresas grades medianas y pequeñas (NOURI; SOLTANI, 2017). Según el tipo de empresa, también existen diferencias en el uso de estrategias de crecimiento, análisis ANSOFF, análisis PEST, análisis de precios, análisis del ciclo de vida del producto, alianzas estratégicas y análisis de cadenas de suministros entre empresas públicas, privadas y mixtas (NOURI; SOLTANI, 2017).

Aunque no todas las técnicas o herramientas son necesarias en una empresa, algunas de ellas son comúnmente usadas en más de un área o en más de un proceso dentro de la empresa; por ejemplo: diagrama de causa efecto, diagramas de Pareto, o histogramas (AHMED; HASSAN, 2003). Para el caso de actividades específicas de gestión de la tecnología las tres herramientas más usadas son el análisis de mercado, herramientas de creatividad y benchmarking (CETINDAMAR; WASTI; BEYHAN, 2012).

La mayoría de estudios apoyan el uso de las herramientas de gestión como parte importante dentro del proceso de planificación estratégica (QEHAJA, 2017). Sin embargo, los países/empresas no utilizan las mismas herramientas; por ejemplo, CUSAT y análisis "What if" son mínimamente utilizadas en países en desarrollo (QEHAJA, 2017). Según Qehaja (2017) las herramientas gerenciales son más utilizadas en países desarrollados, le siguen los países en desarrollo y en última posición las economías en transición.

Tales herramientas no darán beneficios de inmediato, para alcanzar los resultados esperados se requiere un periodo de maduración y estabilidad. Phaal et al. (2006) identifican tres fases para que una técnica llegue a un estado maduro de funcionamiento: a) Fase exploratoria, mediante pruebas iniciales con aplicaciones prácticas, para probar su utilidad y asegurar que se capturan las dimensiones clave del problema; b) Fase de desarrollo, modificando la herramienta con aplicaciones prácticas, y evaluando su utilidad en cada paso; y c) Fase de pruebas, garantiza que las herramientas sean estables y útiles en diferentes escenarios, sin que existan cambios significativos en su funcionamiento.

Así, las organizaciones actuales basan sus modelos gerenciales en estrategias de gestión para direccionar, desarrollar y mantener los procesos haciendo que la organización promueva, mantenga o impulse su efectividad. La terminología de lo que es un modelo es muy amplia, podría tratarse de algo que puede imitar y considerarse como referencia, es un proceso o esquema de trabajo que tiene como finalidad organizar una actividad. García \& Rodríguez (2015) consideran que ninguna herramienta 
gerencial por sí misma es suficiente para direccionar una organización, es necesario también la creatividad y de la habilidad de la gerencia al momento de su aplicación, con el fin de alcanzar un alto desempeño en el desarrollo del capital humano por medio de la identificación, adquisición, transformación y consolidación del conocimiento.

\subsection{BENEFICIOS DE LA ADOPCIÓN DE HERRAMIENTAS GERENCIALES}

Debido a la creciente competitividad, el cambio tecnológico y la globalización, las organizaciones tienen que adaptarse rápidamente a los requerimientos del mercado; con la idea de ser más dinámicas y competitivas; caso contrario, se enfrentan con dificultades y problemas que a la larga ocasionan su extinción (AFONINA; CHALUPSKY, 2012). La necesidad de incorporar herramientas de gestión en las empresas para responder a las necesidades del entorno, es impulsada no sólo por las características de la herramienta, sino también por el contexto en el que se encuentren.

Entre los factores motivadores para el uso de herramientas gerenciales para Ortiz (2006) se destacan: mercado, Iniciativa personal, problemas con las materias primas, deficiencias de calidad, dificultades con los equipos, problemas de contaminación, fallas de seguridad. En ese sentido Ortiz (2006) también señala que el mercado ha sido identificado como el primer factor motivador en la mayoría de negocios y destaca la importancia de las interrelaciones entre las áreas funcionales de mercadeo y producción, por tanto mientras más débil sea el conocimiento y la relación con los clientes por parte de la empresa, menor será su propensión a realizar actividades de cambio o de innovación.

Existen diversas técnicas, herramientas, métodos, modelos, frameworks, enfoques y metodologías de apoyo en la toma de decisiones; estas herramientas son diseñadas y desarrolladas para ser aplicadas en todas las etapas del proceso de gestión estratégica con el objetivo de obtener el mejor desempeño y eficiencia organizacional (QEHAJA; KUTLLOVCI; PULA, 2017). Las Empresas usan herramientas de gestión para mejorar sus actuales procesos, productos, servicios, y para identificar y desarrollar nuevas oportunidades de negocio (BOIKANYO; LOTRIET; BUYS, 2016). Sin embargo, la condición fundamental para que la herramienta adoptada proporcione los beneficios esperados, es que los gerentes tengan un claro entendimiento de para qué sirve ese modelo.

Varios autores destacan los beneficios que las herramientas y técnicas de gestión proporcionan a los gerentes. Afonina \& Chalupsky (2012) las describe en función de los beneficios proporcionados: 1) ayudan a los gerentes a ver los portafolios de negocios de la empresa; 2) consiguen realizar el análisis de la situación del mercado y predecir el desarrollo futuro de la compañía. Para Knott (2006), las herramientas y estrategias son una guía para reflexionar y debatir; además permiten analizar situaciones complejas y ambiguas. Según Stenfors, Tanner, \& Haapalinna (2004) las herramientas gerenciales ayudan a los 
ejecutivos para la toma de decisiones, analizar el entorno, comprender las relaciones, proyectar el futuro, y encontrar soluciones a los problemas de gestión. Knott (2006) explica que a través del uso de las herramienta de gestión se obtienen beneficios como el desarrollo del pensamiento, interacción y el debate que centrados en la necesidad de los usuarios conllevan a la toma de decisiones (STENFORS; TANNER; HAAPALINNA, 2004).

Para Frost (2003) los beneficios de la utilización de técnicas y herramientas de gestión estratégica son: aumentar la conciencia acerca del impacto ambiental del negocio, resolver problemas estratégicos, reducir el riesgo relacionado a la toma de decisiones, establecer prioridades en empresas grandes y complejas, y proporcionar de una marco para evaluar la importancia relativa de diferentes oportunidades de negocios. Gerentes y profesionales, que basan sus decisiones en información obtenida por medio del uso de herramientas gerenciales, son capaces de desarrollar procesos de mejora continua (FANELLI; LANZA; ZANGRANDI, 2017). Según Nouri \& Soltani (2017) el mayor uso de herramientas gerenciales haría que las empresas experimenten un mejor desempeño; mostrando una relación positiva y significativa entre el uso de herramientas y el desempeño organizacional.

No existe una valoración mayor o menor sobre que instrumento aplicar, antes de optar por tal o cual herramienta, los administradores se deben preguntar ¿qué herramienta es la mejor para superar los problemas que enfrenta la organización? La respuesta a esta pregunta depende del tipo de problema al que la organización se enfrenta, así como de las condiciones internes y externas que la gobiernan (NOURl; SOLTANI, 2017). Los gerentes y los tomadores de decisiones primero deben identificar y clasificar sus problemas; segundo, estudiar las funciones y capacidades de las herramientas de gestión; y finalmente, seleccionar y utilizar la herramienta o herramientas adecuadas (NOURI; SOLTANI, 2017). Para que esta selección sea la adecuada, los directivos deben estar más basados en el conocimiento (knowledge-based) para poder clasificar las herramientas, y seleccionar y elegir la adecuada para la empresa (NOURI; SOLTANI, 2017).

\subsection{LAS BARRERAS PARA SU IMPLEMENTACIÓN}

Aunque los gerentes hayan identificado las herramientas que necesitan y sus colaboradores estén dispuestos a implementarlas y utilizarlas, la empresa necesita cierto tipo de capacidades para poder adoptar la herramienta seleccionada. Según Ahmed \& Hassan (2003), antes de la implementación las empresas se enfrentan con barreras que deben ser eliminadas tales como: (1) falta de conocimiento o falta de una visión clara respecto a qué tipo de entrenamiento es necesario;( 2) falta de instalaciones para llevar a cabo un programa de capacitación eficaz, (3) falta de disponibilidad de los empleados para capacitarse debido a que no pueden ausentarse de su lugar de trabajo por periodos de tiempo prolongados; ( 4) falta 
de recursos y apoyo de la alta gerencia; (5) falta de conocimiento estadístico. Cetindamar et al. (2012a) consideran que las empresas sin un nivel apropiado de conocimientos y habilidades no podrán explotar todos los beneficios que le proporcionan las herramientas de gestión.

El nivel de experiencia y la juventud son un factor importante en la apropiación de las herramientas en la empresa. Para Cetindamar et al. (2012a), gestores con menos experiencia y más jóvenes, o que hayan trabajado en diferentes empresas podrían estar más abiertos al uso de herramientas de gestión; lo que también puede significar que los gerentes que son nuevos en la empresa están más motivados para introducir nuevas herramientas y técnicas. Phaal et al. (2006) consideran que las herramientas para ser implementadas deben cumplir con tres condiciones: (a) ser robustas -teóricamente sólidas y confiables-; (b) económicas y de fácil implementación -no demasiado complejas o que requieren muchos recursos-; y (c) integradas -que puedan enlazarse con otros procesos y herramientas ya implementados en la empresa-; y (d) flexibles - adaptarse al contexto particular, en términos de propósito comercial, entorno de mercado, recursos e información disponibles, y cultura corporativa.

Según Cetindamar et al. (2012a) existen otros condicionantes que afectan el éxito en la implementación como la formación educativa en áreas de tecnología, el nivel jerárquico, los años de experiencia en la empresa, y el nivel de compromiso con ella. No es suficiente con que las herramientas de gestión presenten información útil, esta necesita ser reconocida, compartida y formar parte de las actividades profesionales del día a día (FANELLI; LANZA; ZANGRANDI, 2017). En tal sentido, las empresas deben tener condiciones para que las técnicas adoptadas den un beneficio; no basta con su sola aplicación; sí los gerentes y colaboradores no creen en su utilidad y la mejora en sus actividades diarias.

Tomando en cuenta que en los últimos años el aumento de la competencia global ha intensificado los desafíos que enfrentan los gerentes (PÉREZ-MÉNDEZ; MACHADO-CABEZAS, 2015); las herramientas y técnicas son esenciales para gestionar profesionalmente cualquier empresa de fabricación, pequeña, mediana o grande (AHMED; HASSAN, 2003). Sin embargo, la mayoría de estos estudios se han llevado a cabo hasta ahora únicamente en grandes industrias (AHMED; HASSAN, 2003). Lo confirman Nouri \& Soltani (2017) cuando mencionan que el uso de algunas herramientas requiere ingentes recursos financieros y solo pueden ser utilizadas por las compañías más grandes.

En un mundo complejo las organizaciones deben tender a la simplicidad, evitando que la globalización dé lugar a configuraciones corporativas enormemente complicadas plagadas de niveles excesivos de gestión, toma de decisiones difusas, estructuras matriciales difíciles y un aumento exponencial de las comunicaciones, todo lo cual socava el crecimiento y los beneficios (RIGBY; BILODEAU, 2015).

Cetindamar et al. (2012a) mencionan que existe confusión en la definición, lista de herramientas y la teoría bibliográfica, además no hay una descripción y discusión claras sobre las metodologías, herramientas 
y técnicas publicadas. La falta de una recopilación sistemática de listas de herramientas hace que estas se vuelvan poco operativas, la gestión estratégica a menudo ha sido criticada con el argumento de que se basa en principios teóricos y no en las realidades de la gestión y justamente las limitaciones del estudio de herramientas gerenciales está relacionado con los fundamentos y bases teóricas (CETINDAMAR; WASTI; BEYHAN, 2012; NOURI; SOLTANI, 2017; QEHAJA; KUTLLOVCI; PULA, 2017). O si el uso indebido en los casos donde los fundamentos teóricos de la herramienta no son claros, o si los conocimientos y habilidades requeridos para su aplicación efectiva son inadecuados (PHAAL; FARRUKH; PROBERT, 2006). Para Nouri \& Soltani (2017), de hecho muchos escritores de gestión estratégica, han expresado que se pueden usar diferentes tipos de estas herramientas según la situación; sin embargo, como la naturaleza de su función no es clara y no existe una guía en esta área, muchas organizaciones han tenido problemas al utilizar técnicas de planificación estratégica y han sufrido pérdidas significativas. El uso y la comprensión de las ideas de gestión son bastante diferentes en la literatura y la práctica empresarial (NEDELKO; POTOCAN; DABIĆ, 2015).

Para definir una serie de cuestiones clave relacionadas con herramientas de gestión, en primer lugar, se necesitan herramientas prácticas para respaldar decisiones y acciones de gestión, junto con técnicas para su aplicación. En segundo lugar, se requieren procesos de gestión para combinar herramientas y técnicas para abordar problemas específicos. Finalmente, se necesitan marcos conceptuales para guiar la reflexión sobre la gestión, basada en principios teóricos bien fundamentados (PHAAL; FARRUKH; PROBERT, 2006). Nouri \& Soltani (2017) sostienen que las herramientas y técnicas de gestión son poderosas herramientas que ayudan a los gerentes a definir y proporcionar soluciones a los problemas de la organización. Sin embargo, para Phaal et al (2006) el proceso mediante el cual dichas herramientas se aplican en la práctica generalmente requiere algún desarrollo o personalización, lo que puede no ser una tarea sencilla.

Rigby \& Bilodeau (2015) recomiendan elegir las mejores herramientas para el trabajo en las empresas, los gerentes deben tomar una posición muy razonada para seleccionar e implementar herramientas. Una herramienta solo mejorará los resultados en la medida en que identifique a los clientes no satisfechos, se construyan capacidades distintivas, se exploten vulnerabilidades de la competencia y se desarrollen estrategias innovadoras. Adapte las herramientas a su sistema comercial, no al revés. A lo que argumenta Cetindamar et al. (2012a), si con el uso de herramientas gerenciales se pueden medir herramientas y efectividad, podría ser posible desarrollar estrategias para los gerentes sobre cómo mejorar sus habilidades y conocimientos. Presuponemos que las herramientas de gestión utilizadas en las organizaciones dependen de manera importante de las experiencias previas de otros usuarios. Esas experiencias pueden influir de manera importante en la percepción de un empleado sobre la motivación 
para el uso de herramientas de gestión. En consecuencia, esto resulta en una acción del empleado para usar o no usar (NEDELKO; POTOCAN; DABIĆ, 2015).

Pérez-Méndez \& Machado-Cabezas (2015) mencionan que en investigaciones futuras se deberá incluir otras variables no disponibles que posiblemente afecten el sistema de gestión interna de la empresa. Pulgarín \& Molina (2012) aseveran que en la actualidad y desde comienzos del siglo XXI las herramientas de la estrategia se alinean con las nuevas aproximaciones del pensamiento estratégico hacia las turbulencias, los escenarios alejados del equilibrio y, por supuesto, la complejidad. En síntesis, para Qehaja et al. (2017) la competencia global basada en el conocimiento ha creado la necesidad de un trabajo de estrategia útil y procesos efectivos de toma de decisiones.

Para Nedelko et al. (2015) en escenarios del siglo XXI se deben responder varias preguntas básicas, como (1) ¿para qué propósito o trabajo será utilizada la herramienta de gestión?; (2) ¿cuáles son los requisitos y condiciones previas para el uso de herramientas de gestión?; y (3) ¿quién usa herramientas de gestión, para qué trabajo y en qué áreas de trabajo? Phaal et al. (2006) mencionan que un administrador se enfrenta a una serie de desafíos al hacer uso de tales herramientas: ¿cómo encontrar las herramientas adecuadas? ¿Cómo evaluar la calidad y la utilidad de las herramientas disponibles? ¿Cómo aplicar las herramientas en un entorno o proceso práctico? ¿Cómo integrar herramientas con otras herramientas y con procesos y sistemas de negocios?

Qehaja et al. (2017) afirman que las empresas prosperan en el crecimiento y la ventaja competitiva y buscan formas más exitosas de trabajar con el manejo del conocimiento porque los entornos son cada vez más complejos e inciertos, se espera que los gerentes reconozcan y adopten procesos de comprensión más complejos de un mundo cambiante, y como tal, necesitan herramientas y técnicas para una mejor toma de decisiones.

Según Pérez-Méndez \& Machado-Cabezas (2015) no existe un consenso universal sobre qué técnicas constituyen las nuevas herramientas de gestión, sin embargo; la mayoría de los autores consideran como nuevas herramientas no tradicionales: costeo basado en actividades (ABC), gestión basada en la actividad (ABM), cuadro de mando integral (BS), Just In Time (JIT), total gestión de calidad (TQM), cálculo de costes objetivo (TC), contabilidad de gestión estratégica (SMA), costeo del ciclo de vida (LCC), evaluación comparativa y teoría de restricciones (TOC). La prevalencia de estas técnicas indica que las empresas necesitan sistemas de información de gestión (SI) cada vez más precisos y sofisticados que se adapten a las necesidades cambiantes de los gerentes.

Para Rigby \& Bilodeau (2015) un patrón interesante surgió de una clara división entre las regiones que prefieren las herramientas tradicionales y las que buscan nuevas herramientas relacionadas con la tendencia de transformación digital. Tales diferencias pueden ser el resultado de diferentes puntos de 
vista sobre las tendencias: crecimiento e innovación, costo y complejidad, inversión en la transformación digital y una mejor comprensión de los clientes. También es necesario tener en cuenta que los puntajes de satisfacción para la misma herramienta pueden variar ampliamente según el tamaño de la empresa. Por lo tanto, se recomienda que los gerentes de las empresas más pequeñas se centren principalmente en la planificación de escenarios (NOURI; SOLTANI, 2017). Se hace importante armar información relacionada a discutir los resultados por tamaño de empresa, sector y desarrollo a nivel de país (QEHAJA; KUTLLOVCl; PULA, 2017).

Nedelko et al. (2015) afirman que hay evidencia sobre la disminución del uso de algunas herramientas tradicionales. Por ejemplo, la popularidad de la reingeniería de los procesos de negocios ha disminuido en las últimas dos décadas debido a numerosas reorganizaciones infructuosas de los procesos comerciales. También la calidad total la gestión, inicialmente tratada como una fuente potencial de ventaja competitiva sostenible, se ha vuelto menos utilizada cuando su uso no dio como resultado una rentabilidad sostenible. Además, los estudios sobre la implementación de QM / TQM en SMI son relativamente escasos (AHMED; HASSAN, 2003).

Por otro lado, Pulgarín \& Molina (2012) argumentan que aunque las estrategias genéricas son útiles en la definición de posiciones estratégicas para la empresa, así como también en la caracterización de las acciones necesarias para el logro de estrategias, las herramientas de Michael Porter; las disciplinas de Treacy y Wieserma; el análisis de crecimiento del mercado y matriz producto- mercado de Ansoff; el análisis de crecimiento de portafolio y la matriz BCG convergen en el mismo conjunto de carencias porque, generalmente, apuestan a una única posición. En estos casos la organización debe seleccionar una de las posiciones, diseñar una estructura para garantizar su proliferación y ejecutar acciones alrededor de ella que garanticen su sostenimiento.

Aunque el FODA es una de las herramientas de diagnóstico y formulación estratégica más comunes y utilizadas en las empresas, es importante reconocer algunas de sus debilidades. La primera de ellas se relaciona con su incapacidad para analizar situaciones más allá de un momento específico en el tiempo; lo segundo es que la herramienta limita el diagnóstico de factores internos y externos de la empresa a sólo cuatro categorías de variables. Por último, la formulación de estrategias desde la herramienta desconoce las potenciales reacciones del competidor (PULGARINN; MOLINA, 2012). Conforme Phaal et al. (2006), las herramientas basadas en matrices tienen desventajas potenciales, muchos problemas prácticos no pueden ser simplificados a solo dos dimensiones y, por lo tanto, este tipo de herramientas pueden ignorar otros factores importantes.

En un escenario de recursos limitados como es el de la empresa, una apuesta a una estrategia y renuncia a las demás opciones estratégicas posibles representa un riesgo bastante alto para la 
organización; puede darse el caso de que un diagnóstico no correcto y, por supuesto, la estrategia seleccionada tampoco, derivando en un fracaso que acabaría con la empresa a corto plazo (PULGARÍN; MOLINA, 2012). Pérez-Méndez \& Machado-Cabezas (2015) afirman que se debe tomar en cuenta que es muy común la resistencia al cambio entre los usuarios; antecedentes educativos de los empleados; y la necesidad percibida de una gestión más sofisticada. Para Rigby \& Bilodeau (2015), dos obstáculos clave para el crecimiento y los beneficios se vislumbraron importantes: un aumento insidioso de los costos y una complejidad excesiva. Se añade que otra carencia importante está asociada al período de tiempo que para todos los casos es el largo plazo. Las estrategias genéricas procuran acciones en amplios períodos de tiempo para garantizar resultados, lo cual en un entorno cambiante como el de hoy resulta difícil de sostener (PULGARÍN; MOLINA, 2012).

\section{PROCEDIMIENTOS METODOLÓGICOS}

Con el objetivo de determinar los tipos herramientas gerenciales y los beneficios de su adopción en las empresas, fue aplicada una encuesta a 150 micro, pequeñas y medianas o de organizaciones de economía popular y solidaria de los sectores de producción, comercio y servicios de la provincia de Imbabura. La encuesta fue enviada por correo electrónico al representante legal o al gerente de la organización. La recolección de datos se realizó en el periodo de tres meses obteniendo una tasa de respuesta del 40 \% que corresponde a 60 empresas; los datos serán analizados en función de ese número de organizaciones.

El instrumento de recolección de datos fue elaborado en base a la revisión de la literatura y aborda las siguientes variables: a) tipos de herramientas utilizadas, b) en qué áreas de la organización son aplicadas, c) actividades de aplicación, d) barreras de implementación y e) beneficios de su aplicación. El cuestionario consta de 13 preguntas, elaboradas con escala tipo Likert de cinco puntos (1 menos importante y 5 más importante) y otras de opción múltiple. Para el análisis de los datos se aplicó estadística descriptiva y los resultados serán presentados comparando las organizaciones por su tamaño en función al personal ocupado.

\section{RESULTADOS Y ANÁLISIS}

La literatura sobre herramientas de gestión aborda todo tipo de empresas, grandes, medianas y pequeñas, en varios ámbitos: comercio, industriales, servicios, minería; algunas investigaciones hacen énfasis en empresas de un solo sector Boycanyo et al., (2016) y otras se refieren a una región específica Pawliczek et al., (2015). La investigación abarcó empresas de distintos tamaños y sectores con el fin de 


\section{Gestãoe \\ Desenvolvimento}

e-ISSN: 2446-6875

p-ISSN: $1807-5436$

identificar las principales herramientas de gestión utilizadas. De esta manera, fue posible determinar de forma más amplia las tendencias de implementación de herramientas gerenciales en relación al nivel socio-económico de otros países, el tamaño de la empresa, su ámbito de acción, e incluso los beneficios y dificultades que se presentaban.

En relación a las herramientas utilizadas en las empresas, las más usadas son el análisis financiero, la planificación estratégica, la lluvia de ideas, el análisis FODA y las estrategias de crecimiento (Figura 2). Estos resultados difieren de los encontrados por Nedelko et al. (2015) donde en empresas eslovacas las herramientas más usadas son el outsourcing, el benchmarking, las core competencies, y la gestión del conocimiento; en tanto que en empresas croatas son el establecimiento de la misión y visión, el benchmarking, las core competencies, y el CRM. Sin embargo, coincide con lo mencionado por Nouri \& Soltani (2017) quienes afirman que la herramienta más popular en América del Sur es la planificación estratégica. De igual forma, Qehaja et al. (2017) encontraron que las herramientas más usadas en pequeñas y medianas empresas de países en desarrollo son el análisis financiero del negocio, el análisis FODA, el análisis PEST, el benchmarking, y el análisis de factores críticos de éxito. Se observa que solo el análisis FODA se encuentra como una de las herramientas más utilizadas por empresas medianas y pequeñas; como lo afirman Qehaja et al. (2017) en todos los tipos de empresas tanto de países desarrollados como en desarrollo. El uso de la herramienta análisis financiero del negocio es ligeramente superior a la planificación estratégica en empresas grandes, y es igual en medianas y pequeñas. La lluvia de ideas, utilizada en todas las empresas de nuestra investigación, no consta entre las más utilizadas según Qehaja et al. (2017b, 2017a). El Análisis de Porter y benchmarking, herramientas de uso frecuente en todos los países, aparentemente son estrategias de menor aplicación en las empresas de economía popular y solidaria. Las herramientas CUSAT y What if no constan entre las estrategias utilizadas por las empresas encuestas, lo cual coincide con la afirmación de Qehaja; Kutllovci; Pula (2017b) al señalar que son de uso mínimo en países en desarrollo. 


\section{Gestãoe \\ Desenvolvimento}

e-ISSN: 2446-6875

p-ISSN: $1807-5436$

Figura 2. Herramientas utilizadas por las empresas*

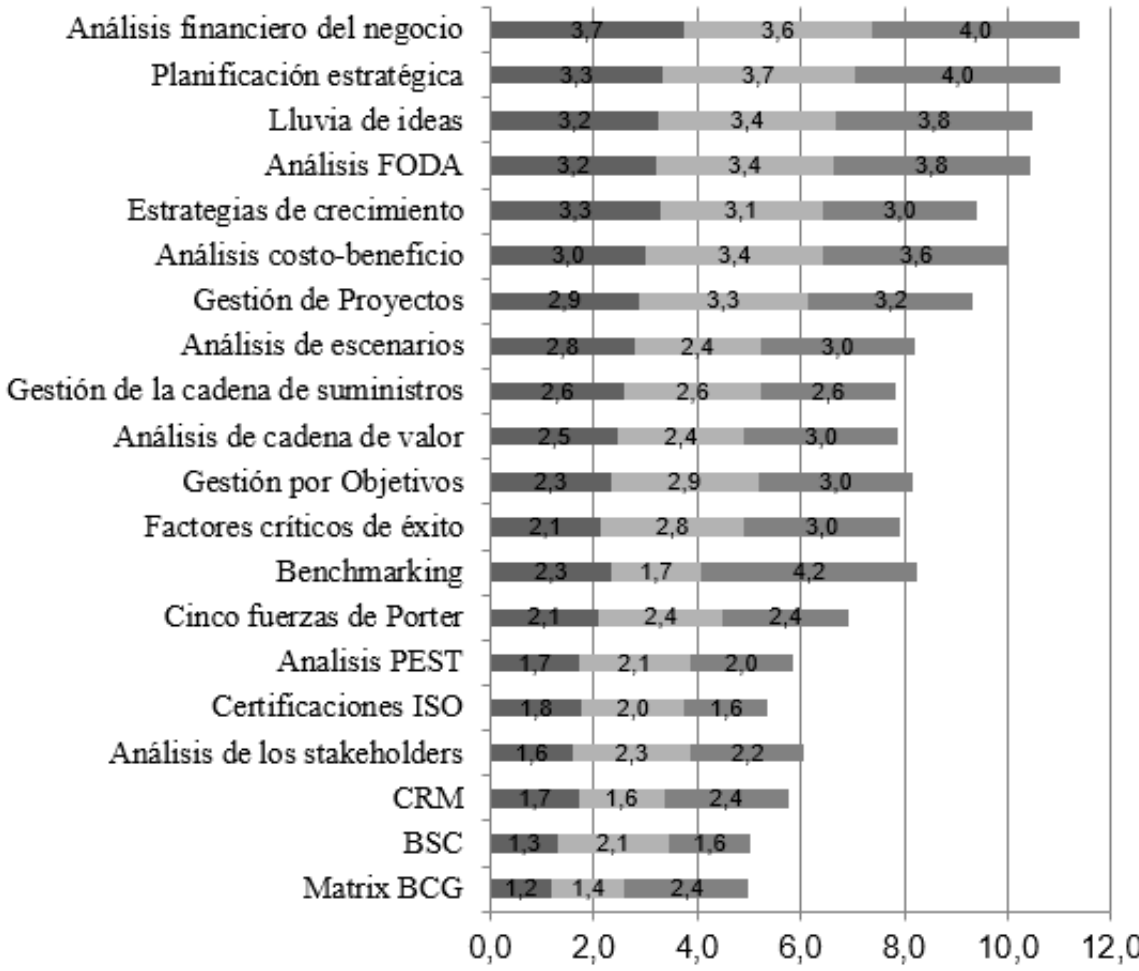

- MICRO

- PEQUENA

MEDIANA

\section{*Escala Likert: 1 Nunca ha utilizado, 2 Utilizada una vez o está considerando utilizar, 3 Utilizada ocasionalmente, 4} Utilizada regularmente, 5 Parte de la política organizacional o procedimiento operativo estándar.

Se observa que la matriz $B C S$, el cuadro de mando integral (BSC), la gestión del relacionamiento con el cliente (CRM), el análisis de los stakeholders, y las certificaciones ISO se posicionan como las menos usadas por las empresas encuestadas.

Respecto al grado de utilización de la herramienta, ninguna de ellas es considerada parte de la política organizacional o procedimiento operativo estándar; incluso las de mayor puntaje solo se utilizan ocasional o regularmente. Al comparar los resultados por tamaño de empresa, se observa una tendencia similar en la apropiación de las herramientas; sin embargo, las empresas de medio porte son las que mayor puntaje general alcanzan. Este resultado podría indicar que a mayor tamaño de la empresa se utiliza una mayor variedad de herramientas; acorde con Cetindamar et al. (2012a) el tamaño de la empresa es positivamente asociado con el número de herramientas y técnicas usadas por las mismas. En esa misma línea los autores establecen una relación positiva entre el número total de herramientas y técnicas utilizadas por una empresa y su éxito para alcanzar su crecimiento. En promedio empresas grandes usan 


\section{Gestãoe \\ Desenvolvimento}

e-ISSN: 2446-6875

p-ISSN: $1807-5436$

8,1 herramientas, medianas 7,6 y pequeñas 5,3 (QEHAJA, 2017; QEHAJA; KUTLLOVCl; PULA, 2017); es decir, el promedio de uso de técnicas y herramientas varia en empresas pequeñas, medianas y grandes (NOURI; SOLTANI, 2017)

Al comparar las Top 10 herramientas más utilizadas del estudio realizado por Qehaja et al. (2017) con los datos obtenidos en esta investigación (Tabla 3) se identificó lo siguiente: a) Si bien el análisis FODA está dentro de Top 10, para las empresas ecuatorianas no es la principal herramienta para su gestión; b) No existe ninguna herramienta que ocupa la misma posición en los dos estudios; c) Solo cinco herramientas -Análisis financiero del negocio, Planificación estratégica, Análisis FODA, Análisis costo-beneficio, Análisis de escenarios- son las mismas en ambas investigaciones, pero en rankings diferentes.

Tabla 3. Top 10 herramientas de gestión utilizadas en PYMES

\begin{tabular}{ccc}
\hline Posición & Resultados obtenidos & Qehaja et al., $\mathbf{2 0 1 7}$ \\
1ra & Análisis financiero del negocio & Análisis FODA \\
2da & Planificación estratégica & Análisis PEST \\
3ra & Lluvia de ideas & Presupuestos \\
$4 \mathrm{ta}$ & Análisis FODA & Benchmarking \\
$5 \mathrm{ta}$ & Estrategias de crecimiento & Establecimiento Misión y visión \\
$6 \mathrm{ta}$ & Análisis costo-beneficio & Análisis financiero del negocio \\
$7 \mathrm{ma}$ & Gestión de Proyectos & Planificación estratégica \\
$8 \mathrm{va}$ & Análisis de escenarios & Estrategias de negocio \\
9na & Gestión de la cadena de suministros & Escenarios pesimista y optimista \\
$10 \mathrm{ma}$ & Análisis de cadena de valor & Análisis costo-beneficio \\
\hline
\end{tabular}

La Figura 3 presenta las empresas que utilizan herramientas de gestión y en qué áreas las aplican. Primero, se observa que las empresas utilizan herramientas de gestión en todas las áreas organizacionales; sin embargo, los resultados no reflejan que exista un área en que todas las empresas apliquen herramientas gerenciales. Segundo, en las áreas comercial y administrativa son donde más las empresas se apalancan con herramientas, aproximadamente siete de cada 10 empresas aplican herramientas en esas dos áreas. Tercero, los datos muestran que en el área de recursos humanos es en donde menos se aplican las herramientas, solo el $35 \%$ de las empresas encuestadas utiliza algún tipo de herramienta para ese departamento. De forma global se podría decir que aproximadamente el $56 \%$ de las empresas analizadas (cinco de cada 10) utiliza herramientas gerenciales en todas las áreas de la organización. 


\section{Gestãoe \\ Desenvolvimento}

e-ISSN: 2446-6875

p-ISSN: 1807-5436

Figura 3. Áreas en que se utilizan las herramientas de gestión

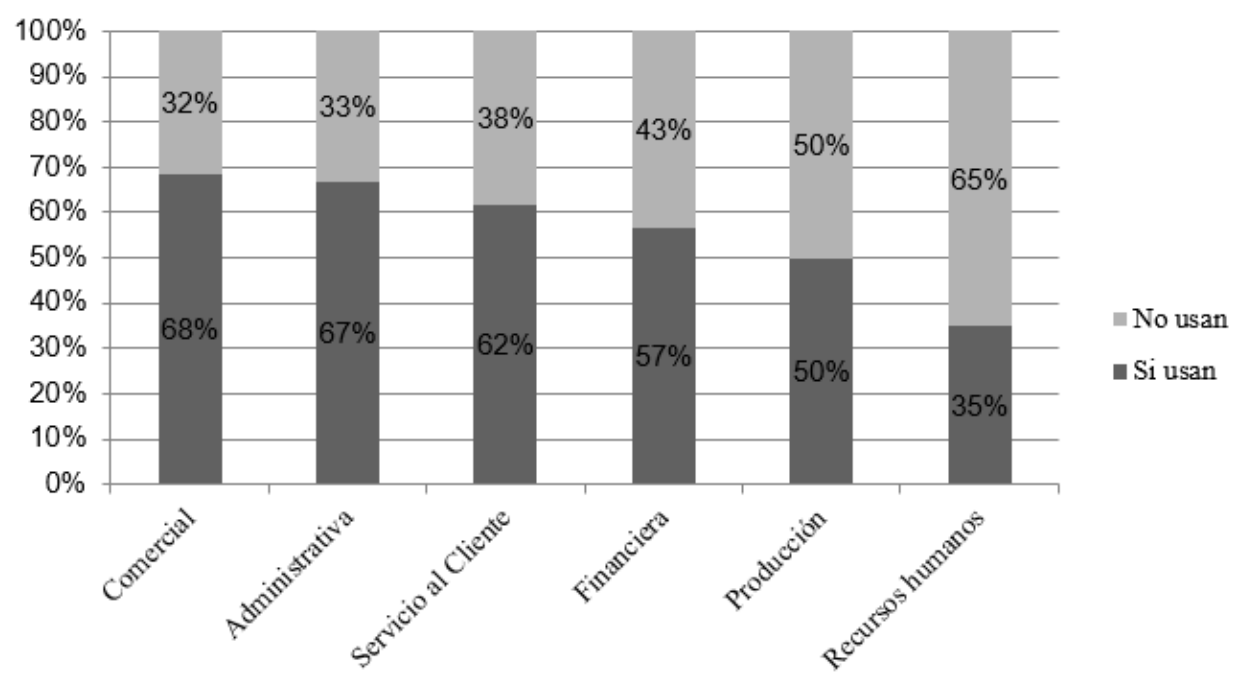

Analizando solo las organizaciones que usan herramientas de gestión, el $75 \%$ de las herramientas con que cuentan son usadas en cuatro áreas - comercial, administrativa, servicio al cliente y financiera (Figura 4). Los datos muestran un equilibrio en la distribución de herramientas por área, que en promedio es de dos herramientas por área organizacional. No obstante, solo en el área de recursos humanos las organizaciones utilizan únicamente una herramienta. 


\section{Gestãoe \\ Desenvolvimento}

e-ISSN: 2446-6875

p-ISSN: 1807-5436

Figura 4. Áreas en que se utilizan las herramientas de gestión

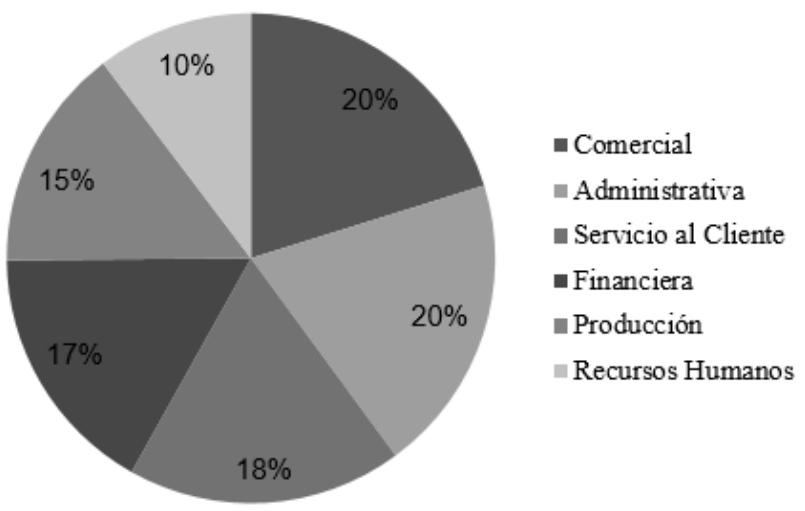

Referente a para qué actividades son utilizadas las herramientas, más de la mitad de las organizaciones encuestadas las aplican para gestionar actividades relacionadas a productividad y eficiencia, y gestión financiera (Figura 5). En contraste, los datos muestran que aproximadamente solo el 25 \% de las organizaciones aplican herramientas para el desarrollo de actividades de producción, gestión de recursos humanos, y gestión de la ciencia y la tecnología. Esto contradice con lo afirmado por Cetindamar et al. (2012) quienes mencionan que el área de I+D es donde más herramientas se utiliza.

Figura 5. Actividades en que se utilizan las herramientas de gestión

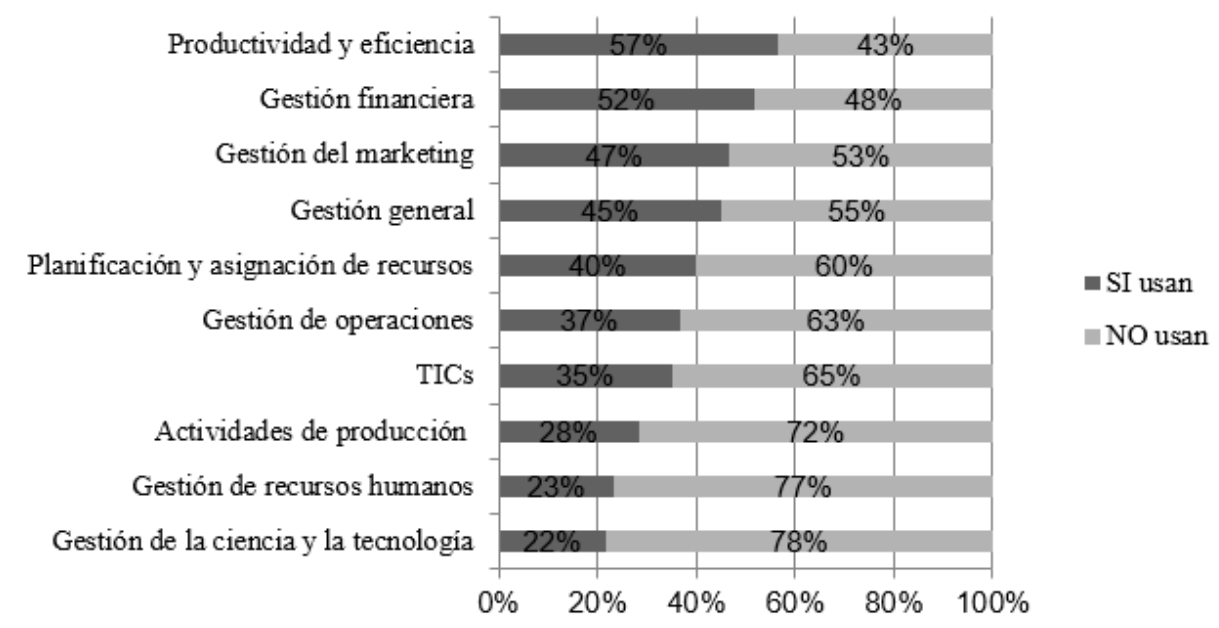




\section{Gestãoe \\ Desenvolvimento}

e-ISSN: 2446-6875

p-ISSN: $1807-5436$

La Figura 6 presenta que el $80 \%$ de las herramientas aplicadas en las organizaciones son dirigidas para desarrollar y resolver actividades de planificación; gestión general, financiera, marketing, y operaciones; y productividad y eficiencia.

Figura 6. Actividades en que se utilizan las herramientas de gestión

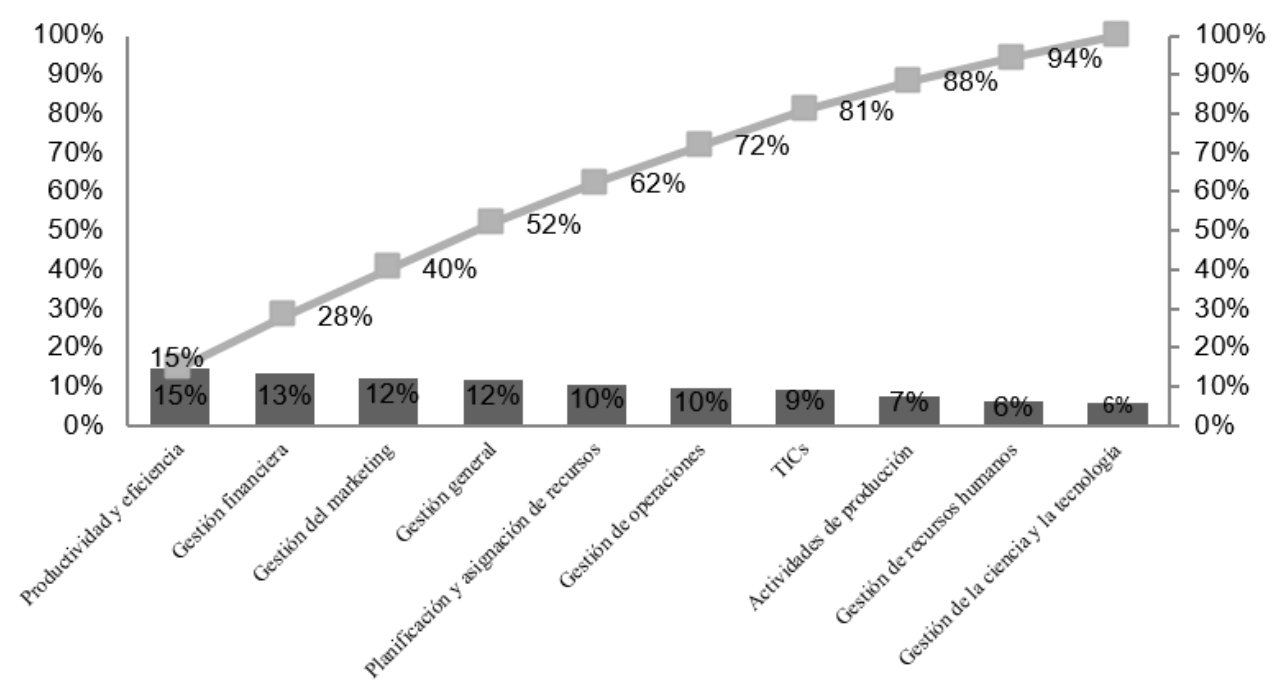

En el momento de implementación de cualquier herramienta de gestión las organizaciones afrontan dificultades. Según las organizaciones encuestadas los principales problemas son de tipo financiero al no contar con los recursos necesarios; disponibilidad de tiempo y recursos para capacitar a sus empleados, debido a que existen puestos de trabajo que no pueden dejar de funcionar; conocimiento uso y adaptabilidad a nuevas tecnologías; y disponibilidad de infraestructura. Estas barreras se presentan en igual grado en todas las organizaciones analizadas pero con un nivel de dificultad más acentuado en las medianas (Figura 7). Los resultados confirman lo encontrado por Ahmed \& Hassan (2003) que identificaron en pequeñas y medianas empresas de Malasia que las barreras para implementar herramientas gerenciales son la falta de recursos, el apoyo de la alta gerencia, y la falta de conocimientos en estadística. De igual manera, coincide con lo afirmado por Nouri \& Soltani (2017) cuando mencionan que el uso de algunas herramientas demanda un alto nivel de recursos financieros las organizaciones deben afrontar cambios fundamentales en su estructura, valores y misión. 


\section{Gestãoe \\ Desenvolvimento}

e-ISSN: 2446-6875

p-ISSN: $1807-5436$

Figura 7. Dificultades en la implementación de herramientas*

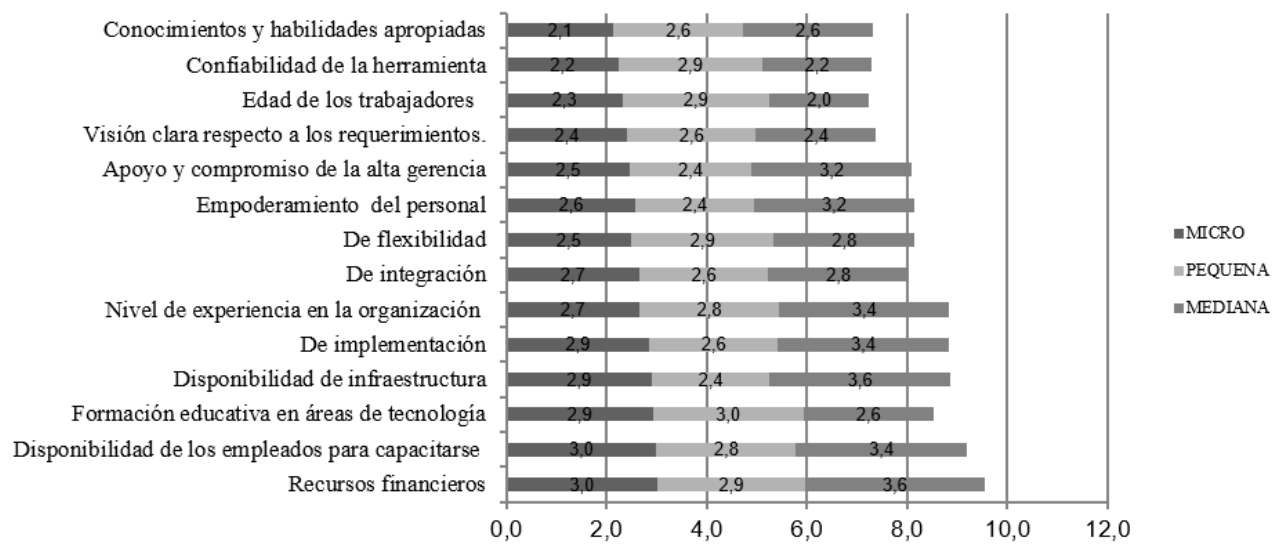

*Escala Likert: 1 No hubo dificultad, 2 Dificultad baja, 3 Dificultad moderada, 4 Dificultad media, 5 Dificultad alta.

Para las organizaciones encuestadas la adopción de herramientas gerenciales ha proporcionado beneficios moderados, independientemente del tamaño de la empresa (Figura 8). Los mayores beneficios están en mejora de productos y procesos, calidad y mejora continua, toma de decisiones estratégicas y desarrollo de nuevas oportunidades de negocio. Como lo corrobora Ahmed \& Hassan (2003) el uso de las herramientas y técnicas de gestión facilita la toma de decisiones objetivas. Los beneficios reportados por las empresas coinciden con lo afirmado por Ortiz (2006) cuando menciona que el mercado, reflejado en el posicionamiento competitivo, lealtad e incremento de clientes, e identificación de nuevas oportunidades de negocios, es el mayor factor de motivación para implementar herramientas gerenciales; y por Afonina \& Chalupsky (2012) al señalar que las empresas necesitan adaptarse a los nuevos requerimientos del mercado. Sin embargo, muchas veces las empresas del sector de economía popular y solidaria trabajan con poco margen de ganancia puesto que su objetivo es social - económico y al no tener inversiones muy altas, la reducción de costos sería la estrategia principal para mantenerse en el mercado (DE SOUZA LESSA et al., 2017). A medida que las empresas reduzcan o eviten los impactos negativos (GOMES JÚNIOR et al., 2020) producto de utilizar adecuadamente las herramientas gerenciales serán las sociedades que conviven con las empresas las más beneficiadas. 


\section{Gestãoe \\ Desenvolvimento}

e-ISSN: 2446-6875

p-ISSN: $1807-5436$

Figura 8. Beneficios obtenidos de las herramientas*

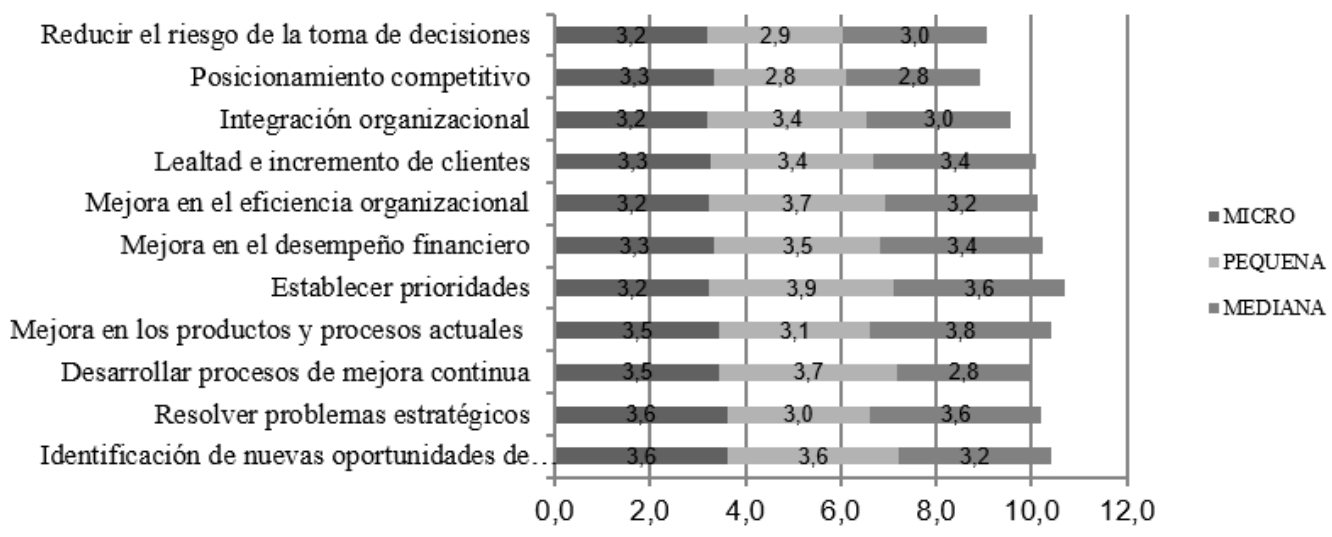

*Escala Likert: 1 No hubo beneficio, 2 Beneficio bajo, 3 Beneficio moderado, 4 Beneficio medio, 5 Beneficio alto

\section{CONSIDERACIONES FINALES}

El objetivo de esta investigación fue determinar los tipos herramientas gerenciales, los beneficios de su adopción y las dificultades encontradas en su implementación en PYMES de la economía popular y solidaria. En relación a cuáles son las herramientas utilizadas, si bien la teoría afirma que el análisis FODA es la herramienta más utilizada, los datos demostraron el interés que poseen las organizaciones por la planificación a través del uso del análisis financiero, la planificación estratégica, la lluvia de ideas, el análisis FODA y las estrategias de crecimiento; sin embargo, restan atención en la satisfacción del cliente. Esto indica que no siempre las herramientas de tendencia son las más utilizadas; la aplicación de tal o cual herramienta dependerá de distintos factores como la región, la cultura, y el entorno socio-económico de la organización. Herramientas que implican un manejo estadístico y matemático de mayor nivel no han sido aplicadas por las empresas encuestadas, posiblemente por desconocimiento técnico de los gerentes o recursos limitados de las empresas.

Respecto a en qué áreas organizacionales son utilizadas, se identificó que las herramientas se emplean en toda la organización. La diferencia en su aplicación radica en las actividades para las cuales son utilizadas y los objetivos que se espera conseguir. Sin embargo, el área de Recursos Humanos es la que menos herramientas utiliza para su gestión.

La investigación confirma que la aplicación de instrumentos que permitan mejorar la gestión en las organizaciones se orienta más a actividades administrativas que a aspectos de producción, innovación o 
capacitación de empleados. Esto puede ocurrir porque las organizaciones con bajos montos de inversión entran en un mercado de alta competencia que demanda una estructura organizativa para acceder a créditos y lograr un posicionamiento positivo en el mercado.

Si bien entre las empresas encuestadas algunas se dedican a actividades de producción no son industrias grandes, lo que explica el menor uso de herramientas de costeo, indispensables para volúmenes grandes de insumos.

Las empresas de economía popular y solidaria, en Ecuador -por lo general-, tienen una trayectoria de vida de pocos años, por razones como alta competencia en el mercado, recursos financieros escasos u oferta de productos homogéneos. Esto podría ser un motivo para trabajar con pocas herramientas de gestión ya que los beneficios que proporcionan no son inmediatos y se requiere que la empresa haya logrado posicionarse y tenga reconocimiento. Además, empresas sin niveles apropiados de conocimientos y habilidades se les dificulta aprovechar los beneficios que les podría brindar las herramientas de gestión.

En función de los resultados obtenidos, la implementación de una herramienta gerencial será exitosa, si ésta se adapta a las necesidades y capacidades de la empresa y apoya los objetivos estratégicos. El éxito de la implementación se verá reflejado en nuevas oportunidades de negocio, solución de problemas estratégicos y desarrollo de procesos de mejora continua, entre otros. Con todo, las principales dificultades para implementar herramientas se centran en la falta de recursos financieros, falta de tiempo para capacitación, bajo nivel de conocimientos en áreas de tecnología y disponibilidad de infraestructura.

Es importante a futuro considerar la diferencia de gestión en la utilización de herramientas gerenciales entre Pymes -sean sociedades o personas naturales- y dentro de estas la economía popular y solidaria. Porque herramientas y técnicas son importantes en la gestión de las empresas, sin embargo; no es igual una empresa familiar a una empresa conformada como sociedades que disponen de mayores conocimientos y recursos para gestionarse.

De igual forma, en investigaciones futuras se deberán incluir otras variables no disponibles que posiblemente afecten el sistema de gestión interna de la empresa, prioritario para las empresas trabajar con el manejo del conocimiento porque los entornos son cada vez más complejos e inciertos en un mundo cambiante, y como tal, necesitan herramientas y técnicas para una mejor toma de decisiones. Si bien las herramientas tradicionales como el FODA, análisis PEST, planeación estratégica, entre otras; son las más utilizadas y que la mayoría de los problemas en la toma de decisiones están en la subjetividad humana y la imprecisión del mundo real (BUENO et al., 2020) es necesario pensar en métodos y herramientas para aproximar las incertidumbres de eventos futuros, estas herramientas de estrategia se alinean con las nuevas aproximaciones del pensamiento estratégico hacia las turbulencias, los escenarios alejados del equilibrio y, por supuesto, la complejidad. Sin olvidar que en la complejidad las organizaciones deben 


\section{Gestãoe \\ Desenvolvimento}

e-ISSN: 2446-6875

p-ISSN: $1807-5436$

tender a la simplicidad y hacer que la sociedad sea la beneficiada resultado de un adecuado manejo de herramientas gerenciales.

\section{REFERENCIAS}

AFONINA, Anna; CHALUPSKY, Vladimir. The current strategic management tools and techniques: The evidence from Czech Republic. Economics and Management, [S. I.], v. 17, n. 4, p. 1535-1544, 2012.

AHMED, Shamsuddin; HASSAN, Masjuki. Survey and case investigations on application of quality management tools and techniques in SMIs. [S. I.], 2003.

ARMSTRONG, Michael. A handbook of MANAGEMENT techniques: the best-selling guide to modern management methods. [S. I.]: Kogan Page Publishers, 2001.

ASAMBLEA NACIONAL DEL ECUADOR. LEY ORGANICA DE ECONOMIA POPULAR Y SOLIDARIA Y DEL SECTOR FINANCIERO[S. I.: S. n.]p. 65.

BOIKANYO, Dinko Herman; LOTRIET, Ronnie; BUYS, Pieter W. Investigating the use of business, competitive and marketing intelligence as management tools in the mining industry. Problems and Perspectives in Management, [S. I.], v. 14, n. 2, 2016.

BUENO, Wagner Pietrobelli et al. Fuzzy in Lean To Evaluate the Decision Degree. Revista Gestão e Desenvolvimento, [S. I.], v. 17, p. 4-23, 2020.

CÁCERES, Gladys et al. Herramientas Gerenciales para una mejor Administración Ambiental Aplicables a la Región Andina. Visión Gerencial, [S. I.], v. 3, n. 2, p. 16-24, 2004.

CETINDAMAR, Dilek; WASTI, Nazli; BEYHAN, Berna. Technology Management Tools and Techniques: Factors Affecting Their Usage and Their Impact on Performance. International Journal of Innovation and Technology Management, [S. I.], v. 09, n. 05, p. 1250036, 2012.

DE OLIVEIRA CARLOS, Maria da Graça et al. POLITICAS DE INOVAÇÃO E DETERMINANTES DA INOVATIVIDADE. ESTUDO EM EMPRESAS DA REGIÃO METROPOLITANA DE FORTALEZA. Gestaoe Desenvolvimiento, [S. I.], n. 85, p. 1-19, 2015.

DE SOUZA LESSA, Bruno et al. Innovating for Social Demands - a Double Case Study in Effective Social Enterprises From the Brazilian Semiarid. Revista Gestão e Desenvolvimento, [S. I.], v. 14, n. 2, p. 4, 2017. 


\section{Gestãoe \\ Desenvolvimento}

e-ISSN: 2446-6875

p-ISSN: 1807-5436

FANELLI, Simone; LANZA, Gianluca; ZANGRANDI, Antonello. Management Tools for Quality Performance Improvement in Italian Hospitals. International Journal of Public Administration, [S. I.], v. 40, n. 10, p. 808-819, 2017. Disponivel em: https://doi.org/10.1080/01900692.2017.1280821

FROST, Frederick A. The use of strategic tools by small and medium-sized enterprises: an Australasian study. Strategic Change, [S. I.], v. 12, n. 1, p. 49-62, 2003.

GARCÍA, Marysther; RODRÍGUEZ, María Asunción. Assessment Management. Revista Científica Electrónica de Ciencias Gerenciales, [S. I.], v. núm.30, p. 142-162, 2015.

GOMES JÚNIOR, Alexandre De Araújo et al. Criação De Valor Compartilhado E Negócios Sociais: Explorando Relações Entre Estratégias E Dimensões. Revista Gestão e Desenvolvimento, [S. I.], p. 24-48, 2020.

HABERKAMP, Angela Maria et al. ANÁLISE CRITICA DO NIVELL DE CONHECIMENTO. Revista Gestão e Desenvolvimento, [S. I.], v. 16, p. 152-176, 2019.

JACOME, Estrella et al. EPS, Historias y prácticas de su fortalecimiento. Serie Estudios Economía PopuIar y Solidaria, [S. I.], n. 978-9942-22- 070-7, p. 400, 2016.

KNOTT, Paul. A typology of strategy tool applications. Management Decision, [S. I.], v. 44, n. 8, p. 10901105, 2006.

NEDELKO, Zlatko; POTOCAN, Vojko; DABIĆ, Marina. CURRENT AND FUTURE USE OF MANAGEMENT TOOLS. [S. I.], ก. 1, 2015.

NOURI, Bagher Asgarnezhad; SOLTANI, Milad. Analyzing the use of strategic management tools and techniques between Iranian firms. Academy of Strategic Management Journal, [S. I.], v. 16, n. 1, p. 1-18, 2017.

ORTIZ, F. Gestión de innovación tecnológica en PYMES manufactureras. Congreso Iberoamericano de Ciencia, tecnología, Sociedad e Innovación CTS+ I, [S. I.] v. 12, p. 17, 2006. Disponível em: http://www. oei.es/memoriasctsi/mesa12/m12p22.pdf

PASANEN, Mika. Strategic management tools and techniques in SMEs. In: 2011, Society of Interdisciplinary Business Research (SIBR) 2011 Conference on Interdisciplinary Business Research. [S. I.: S. n.] p. 7.

PAWLICZEK, Adam; MEIXNEROVA, Lucie; NAVRATILOVA, Daniela. Influential analysis of selected management tools on economic value added based on difference analysis method. International Business Management, [S. I.], v. 9, n. 6, p. 1249-1256, 2015. 


\section{Gestãoe \\ Desenvolvimento}

e-ISSN: 2446-6875

p-ISSN: 1807-5436

PÉREZ-MÉNDEZ, José Antonio; MACHADO-CABEZAS, Ángel. Relationship between management information systems and corporate performance. Revista de Contabilidad, [S. I.], v. 18, n. 1, p. 32-43, 2015.

PHAAL, Robert; FARRUKH, Clare J. P.; PROBERT, David R. Technology management tools : concept, development and application. [S. I.], v. 26, p. 336-344, 2006.

PULGARÍN, Sergio; MOLINA, Alberto. Las herramientas estratégicas : un apoyo aL proceso de toma de. [S. I.], v. 10, n. 16, p. 89-114, 2012.

QEHAJA, Albana Berisha. Strategic Management Tools and Techniques : A Comparative Analysis of Empirical Studies. [S. I.], v. 19, n. 1, p. 67-99, 2017.

QEHAJA, Albana Berisha et al. STRATEGIC MANAGEMENT TOOLS AND TECHNIQUES USAGE : A QUALITATIVE REVIEW. [S. I.], v. 65, n. 2, 2017.

QEHAJA, Albana Berisha; KUTLLOVCI, Enver; PULA, Justina. Strategic management tools and techniques usage: A qualitative review. Acta Universitatis Agriculturae et Silviculturae Mendelianae Brunensis, [S. l.], v. 65, n. 2, p. 585-600, 2017.

RIGBY, By Darrell; BILODEAU, Barbara. Management Tools \& Trends 2015. [S. I.], 2015.

STENFORS, Sari; TANNER, Leena; HAAPALINNA, Ilkka. Executive Use of Strategy Tools : Building Shared Understanding through Boundary Objects. Frontiers of E-Business Research, [S. I.], n. January 2004, p. 635-645, 2004. 